Bangladesh J. Bot. 48(4): 1011-1019, 2019 (December)

\title{
RELATIONSHIPS AMONG CULTIVATED PEAS AND THEIR WILD RELATIVES: MOLECULAR AND MORPHOLOGICAL CHARACTERIZATION
}

\author{
Fatih Hanci* and EsRa Cebeci ${ }^{1}$ \\ Erciyes University, Faculty of Agriculture, Melikgazi, Kayseri, Turkey \\ Keywords: Locus, Microsatellite, Morphology, Phenotype
}

\begin{abstract}
This study was conducted to determine relationship between some wild pea accessions (Pisum fulvum L., $P$. abyssinicum L., $P$. sativum var. elatius), local varieties ( $P$. sativum var. sativum $\mathrm{L}$. and $P$. sativum var. arvense L.) and commercial varieties "Boogie" and "Rondo". The genetic diversity was evaluated with 14 simple sequence repeat markers and 50 morphological characters. The results of morphology indicated that, genotypes showed a clustering pattern based on the taxonomic groups when considering only flower characters and all morphological characters. During the molecular study, a total of 48 alleles were obtained. Used all primers showed polymorphism in accessions. The number of alleles varied between $2-6$ among 14 SSR loci revealing the polymorphism level of markers. Similarity coefficient (Dice's) ranged from 0.100 to 0.800 with an average of 0.378 . A dendrogram grouped the 15 genotypes into two main clusters. This information can be utilized for genetic analysis, genotype identification from different sources and development of improved germplasm.
\end{abstract}

\section{Introduction}

The taxonomic classification of Pisum based on karyology and morphology has varied over time. Despite the wide phenotypic and genetic variability, available classifications are still confusing (Korstein and Bogdanova 2008). Davis (1970), Kupicha (1981) recognized species as $P$. fulvum and $P$. sativum. $P$. abyssinicum was not considered as third presumed species by these authors. In addition to these assumptions, Vershinin et al. (2003) reported strong relationships among $P$. humile, $P$. elatius and $P$. sativum. These scientists also grouped the genus into three major classes: $P$. abyssinicum, $P$. fulvum and $P$. sativum- $P$. humile - P. elatius complex. Three species ( $P$. abyssinicum, $P$. fulvum and $P$. sativum) were recognized with two subspecies ssp. sativum and ssp. elatius (Bieb.) by Maxted and Ambrose (2001) in a phylogenetic organization of taxa. According to the actual integrated taxonomic information system database, the Pisum genus has only two species: P. fulvum and P. sativum L. According to same data, the P. sativum $\mathrm{L}$. species has five varieties: P. sativum var. arvense (L.) Poir. (Austrian winter pea), P. sativum var. elatius (Steven ex M. Bieb.) Alef., P. sativum var. macrocarpon Ser., P. sativum var. pumilio Meikle, P. sativum var. sativum L. (garden pea) (ITIS 2018).

This study focused on examining the molecular and morphological features based on plant organs of the 15 Pisum accessions (wild peas, local varieties and commercial varieties). Results of the present study may ensure some beneficial information for the conservation and the use of these Pisum accessions in future breeding programs.

\section{Materials and Methods}

The genotypes of Pisum sativum var. sativum, P. sativum var. arvense, P. sativum var. elatius, $P$. abyssinicum and $P$. fulvum were used as plant material (Table 1).

\footnotetext{
*Author for correspondence: <tanerfatih@gmail.com>. ${ }^{1}$ Bati Akdeniz Agricultural Research Institute, Antalya, Turkey.
} 
Fifteen Pisum accessions were planted on the 16th March 2016, and 23th March 2017. The experiment was designed according to the randomized block design with three replications. Data on different agronomic characters were recorded on individual plant basis from 20 plants randomly selected in each plot appropriately to the International Union for the Protection of New Varieties of Plants (Table 3).

Table 1. List of genotypes used in study and their origins.

\begin{tabular}{lllll}
\hline & Accession name & Geographical origin & Taxonomic name & Resource \\
\hline 1 & 65 PA099 & Ethiophia & P. abysinicum & ARS-USDA, USA \\
2 & 66 PA099 & Yemen & P. abyssinicum & ARS-USDA, USA \\
3 & 67 PF099 & Antalya, Turkey & P. fulvum & ARS-USDA, USA \\
4 & 68 PF099 & South Anatolia, Turkey & P. fulvum & ARS-USDA, USA \\
5 & 69 PSE099 & Denizli, Turkey & P. sativum var. elatius & ARS-USDA, USA \\
6 & 70 PSE099 & Mardin, Turkey & P. sativum var. elatius & ARS-USDA, USA \\
7 & BOOGIE & Com. Variety & P. sativum var. sativum & Commercial var. \\
8 & 75 PSE098 & Mersin, Turkey & P. sativum var. elatius & JIC, UK \\
9 & 105PSC098 & Aydin, Turkey & P. sativum var. arvense & JIC, UK \\
10 & 115 PSA097 & Mardin, Turkey & P. sativum var. arvense & AARI, TR \\
11 & 116 PSA097 & Kastamonu, Turkey & P. sativum var. arvense & AARI, TR \\
12 & 117PS097 & Çorum, Turkey & P. sativum var. sativum & AARI, TR \\
13 & 122PSA097 & Bayburt, Turkey & P. sativum var. arvense & AARI, TR \\
14 & 141 PS097 & Çanakkale, Turkey & P. sativum var. sativum & AARI, TR \\
15 & RONDO & Com. Variety & P. sativum var. sativum & Commercial var. \\
\hline
\end{tabular}

Table 2. Details of the SSR primers used in the study.

\begin{tabular}{lccccc}
\hline SSR marker & Temp. $\left({ }^{\circ} \mathrm{C}\right)$ & Linkage group & Allel size (bp) & No. of alleles & PIC \\
\hline AA122 & 61 & IV & $175-225$ & 4 & 0.833 \\
AA205 & 51 & II & $175-225$ & 2 & 0.154 \\
AA446 & 51 & VII & $450-465$ & 5 & 0.892 \\
AA5 & 61 & III & $225-250$ & 3 & 0.311 \\
AB141 & 61 & III & $175-225$ & 3 & 0.592 \\
AB23 & 61 & V & $200-225$ & 3 & 0.681 \\
AC58 & 61 & V & $200-225$ & 3 & 0.585 \\
AD146 & 51 & VII & $375-425$ & 6 & 0.658 \\
AD147 & 61 & I & $300-325$ & 3 & 0.753 \\
AA67 & 51 & I & $330-390$ & 4 & 0.820 \\
AB72 & 55 & II & $450-500$ & 4 & 0.523 \\
AA175 & 61 & III & $225-250$ & 3 & 0.716 \\
AA285 & 51 & IV & $250-275$ & 3 & 0.574 \\
AB64 & 61 & III & $350-400$ & 2 & 0.611 \\
\hline
\end{tabular}


Table 3. Morphological traits regarding leaf-stem, flower and pod-seed characters.

\begin{tabular}{lll}
\hline Leaf-stem (LS) & Flower (F) & Seed-pod (SP) \\
\hline Length of leaflet (LS1) & Time of flowering (F1) & Shape of seed (SP1) \\
Width of leaflet (LS2) & Maximum number of flowers & Color of cotyledon of seed (SP2) \\
Size of leaflet (LS3) & per node (F2) & Marbling of testa (SP3) \\
Length of stipule (LS4) & Color of wing (F3) & Violet or pink spots on testa \\
Width of stipule (LS5) & Intensity of color of wings (F4) & (SP4) \\
Color of leaflet (LS6) & Intensity of color of standard & Hilum color on seed (SP5) \\
Intensity of color of leaflet (LS7) & (F5) & Color of testa (SP6) \\
Leaflets (absent or present) (LS8) & Color of standard (F6) & Wrinkling of seed cotyledon \\
Waxiness of upper leaflet (LS9) & Width of standard (F7) & (SP7) \\
Dentation of leaflet (LS10) & Shape of base of standard (F8) & Type of starch grains (SP8) \\
Degree of dentation of leaflet (LS11) & Undulation of standard (F9) & Width of seed (SP9) \\
Size of stipule (LS12) & Width of upper sepal (F10) & Curvature on pod (SP10) \\
Shape of stipule (LS13) & Shape of apex of upper sepal & Type of curvature of pod (SP11) \\
Flecking of stipule (LS14) & (F11) & Shape of distal part of \\
Density of flecking of stipule (LS15) & Length of peduncle & pod (SP12) \\
Anthocyanin coloration of stem (LS16) & (up to first flower) (F12) & Color of pod (SP13) \\
Length of plant (LS17) & & Intensity of green color of pod \\
Fasciation of stem (LS18) & & (SP14) \\
Intensity of color of foliage (LS19) & & Anthocyanin coloration \\
Stem length (LS20) & & of parchment (SP15) \\
Number of nodes up to first fertile & & Anthocyanin coloration of \\
Node (LS21) & & pod (SP16) \\
Length from axil to first leaflet or & & \\
tendr (LS22) & & \\
\hline
\end{tabular}

Cluster analysis conducted on the matrix of Euclidean distances generates a dendrogram using the Ward method for each observation group (Ghixari et al. 2014). SAS Institute Inc. JMP ${ }^{\circledR}$ and IBM SPSS ${ }^{\circledR}$ Statistics Ver. 221 were used for all the statistical procedures during morphological characterization studies.

The bulked representative of six individual plants was used for DNA extraction using a MACHEREY-NAGEL NucleoSpin ${ }^{\circledR}$ Plant II kit (MACHEREY-NAGEL GmbH \& Co. KG., Düren, Germany). SSR primer pairs were preferred for high power of discrimination, considering the previous report (Loridon et al. 2005) (Table 2). The PCR was performed in a $25 \mu$ l volume of a master mixture containing $2 \mathrm{mM} \mathrm{MgCl} 2,1 \mathrm{U}$ Taq DNA polymerase (Fermentas, Pittsburgh, PA, USA), 20 - $25 \mathrm{ng}$ genomic DNA, $200 \mu \mathrm{M}$ deoxyribonucleotide triphosphates, $1 \mathrm{X}$ Taq buffer, and $0.6 \mathrm{mM}$ reverse and forward primers (Kumari et al. 2011).

Amplification protocol was 1 cycle of denaturation for $3 \mathrm{~min}$ at $94^{\circ} \mathrm{C}$ (preamplification); 30 sec at $94^{\circ} \mathrm{C}$ for denaturation, $30 \mathrm{sec}$ at $5^{\circ} \mathrm{C}$ for annealing, followed by $1 \mathrm{~min}$ at $72^{\circ} \mathrm{C}$ for extension, for a total of 40 cycles; and the final extension at $72^{\circ} \mathrm{C}$ for $10 \mathrm{~min}$. PCR products were analyzed on 3\% agarose gel stained with ethidium bromide (EtBr) in Tris-borate EDTA (TBE) buffer and 
visualized under UV light (Kumari et al. 2011). Microsatellite bands were scored as either present (1) and absent (0). The values of polymorphic information content (PIC) were obtained using this formula (Hildebrand et al. 1992):

$$
P I C=1-\sum_{i=1}^{n} p_{i}^{2}-2 \sum_{i=1}^{n-1} \sum_{j=t+1}^{n} p_{i}^{2} p_{j}^{2}
$$

The $p_{i}$ and $p_{j}$ represent the population frequency of the $i_{t h}$ and $j_{t h}$ allele, respectively.

Dice's coefficients were used to obtain similarity matrices. To obtain the matrix which was computed with the UPGMA algorithm (unweighted pair group method with arithmetic mean) the XLSTAT program was used (Garcia-Valle et al. 1999).

\section{Results and Discussion}

Allelic variation was clearly observed among the accessions with the used primers. A total of 48 bands were detected by 14 SSR primers, thus amplifying an average of 3,43 bands for each primer. Among them, 47 bands were found polymorphic. The SSR primer AA122 (175 bp) was monomorphic among accessions, therefore, it was not used for further evaluations. Genetic distance between Pisum accessions varied in the range of Dice's similarity coefficient from 0.100 to 0.800 and average distance has been identified as 0.379. Among the accessions, genetic similarity coefficient found 0.800 as the highest value between genotypes 075PSE098 (from Mersin province) and 070PSE098 (from Mardin province). The lowest value was determined between 75PSE098 and "Rondo" (a commercial variety). The dendrogram obtained from the analysis of Pisum accessions is divided into two groups (G1-2) at 30\% genetic distance (Fig. 1).

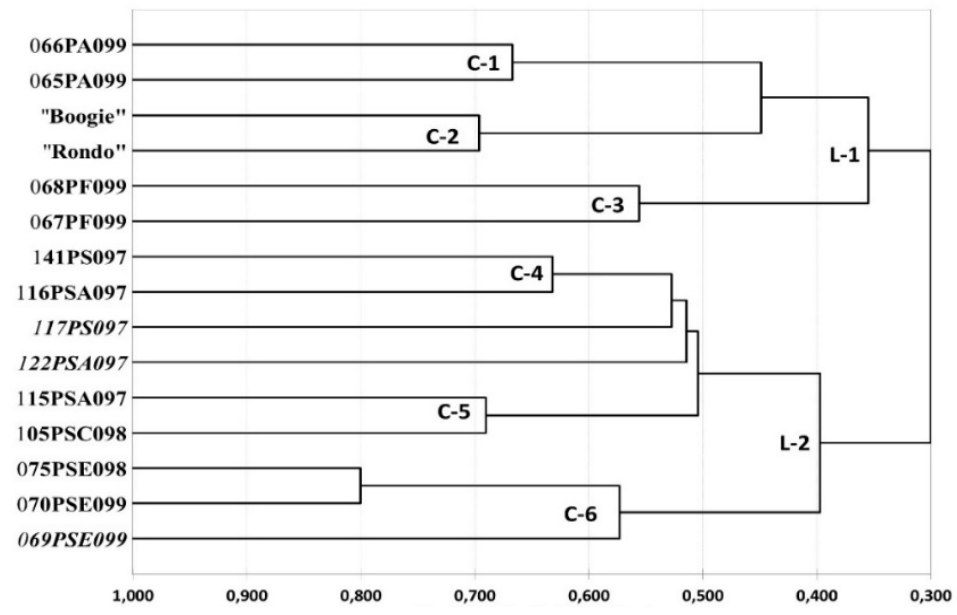

Fig. 1. Cluster analysis of Pisum genotypes based on molecular data.

Group-I is comprised of six accessions, having two commercial varieties. This group includes also two $P$. fulvum L. and two $P$. abyssinicum $\mathrm{L}$. accessions. The combination of cultured peas and wild genotypes was remarkable. Group-II is comprised of nine genotypes, having three P. sativum var. elatius landraces; two local varieties ( $P$. sativum var. sativum L.), four $P$. sativum var. arvense L. accessions. Polymerase chain reaction-based assays have been used to study the genetic 
polymorphism in various plant species. In Pisum species, these SSR primers have made possible the characterization of different accessions, the understanding of phylogenetic relationships, and genetic mapping.

Allele sizes were found similar to expected values (Loridon et al. 2005, Cupic et al. 2009, Kumari et al. 2011). Nisar et al. (2017) reported that an average of 4.69 alleles per SSR locus was obtained in newly developed Pakistani pea lines. Similarly, 2 to 4 alleles per locus were reported in Spanish pea accessions (Martin-Sanz et al. 2011). Using SSR markers, Hagenblad et al. (2014) reported 5 to 10 alleles in the Swedish garden pea. Similarly, allele number per locus averaged 3.1 in the work of Teshome et al. (2015).

PIC values of all studied primers ranged from 0.154 to 0.892 with a mean of 0.622 . PIC values obtained in the present research were higher than the previous studies of the various researchers. Nisar et al. (2017) obtained the maximum PIC value of 0.630 in 23 pea accessions while Kumari et al. (2011) obtained the maximum PIC value of 0.657 in 28 genotypes. This value varied from 0.055 to 0.660 with a mean of 0.460 in the work of Ahmad et al. (2012). In the present study, the high polymorphism values (average PIC, 0.622; maximum PIC, 0.892) is through the efficiency of the preferred SSR primer pairs. Clusters of studied accessions revealed based on molecular data. Only $P$. sativum var. arvense and $P$. sativum var. sativum $\mathrm{L}$. accessions grouped in different clusters (Fig. 1). According to the results of the cluster analysis, it was interesting that the commercial varieties "Rondo" and "Boogie" were distinguished from other $P$. sativum var. sativum accessions (Fig. 1). The high level of genetic diversity obtained among the 15 Pisum genotypes based on Dice's similarity coefficients (ranged from 0.100 to 0.800 ). Samec and Našinec (1996) have reported a narrow diversity $(0.69-0.88)$ between cultivars of $P$. sativum ssp. sativum and $P$. sativum ssp. arvense, whereas a much higher range (0.49-0.98) was obtained between the wild species $P$. sativum ssp. elatius and P. sativum ssp. humile. Ford et al. (2002) reported the largest distance among $P$. sativum and $P$. fulvum accessions.

According to the results of molecular marker application, used microsatellite markers confirmed that the present experiment Pisum accessions have big genetic variability. All 14 primer pairs cross-amplified in specimens of the widespread sister-subspecies $P$. sativum var. arvense, $P$. sativum var. elatius, $P$. abyssinicum, and $P$. fulvum. These molecular markers will be useful for studying genetic diversity and structure as well as for better assessing the conservation status of populations of Pisum. In the future these results can help breeders for interspecific crossing attempts. Also, the application of different marker systems such as ISSR, RAPD etc., may make a significant contribution to the findings obtained in this study.

The cluster analysis was made separately for each agronomic character's group (leaf and stem, flower, seed and pod) for detailed evaluations. In addition, each dendrogram was prepared for both individual Pisum accessions (Figs 2 - 5). The members of groups did not correspond in terms of taxonomic classification based on leaf and stem characters (Fig. 2). Two main clusters were obtained using flower characters for all individual accession (Fig. 3). Members of groups did not correspond in terms of taxonomic classification when considering the flower characters. For example, two $P$. sativum var. elatius accessions were located in two separate groups. When considering the seed-pod characters, the largest distance coefficient was 44.09 between 65PA099 and 67PF099, while the least was 0.01 (between 67PF099 and 68PF099; 65PA099 and 66PA099). Average distance coefficient among all investigated accessions was 13.18 (Fig. 4). 


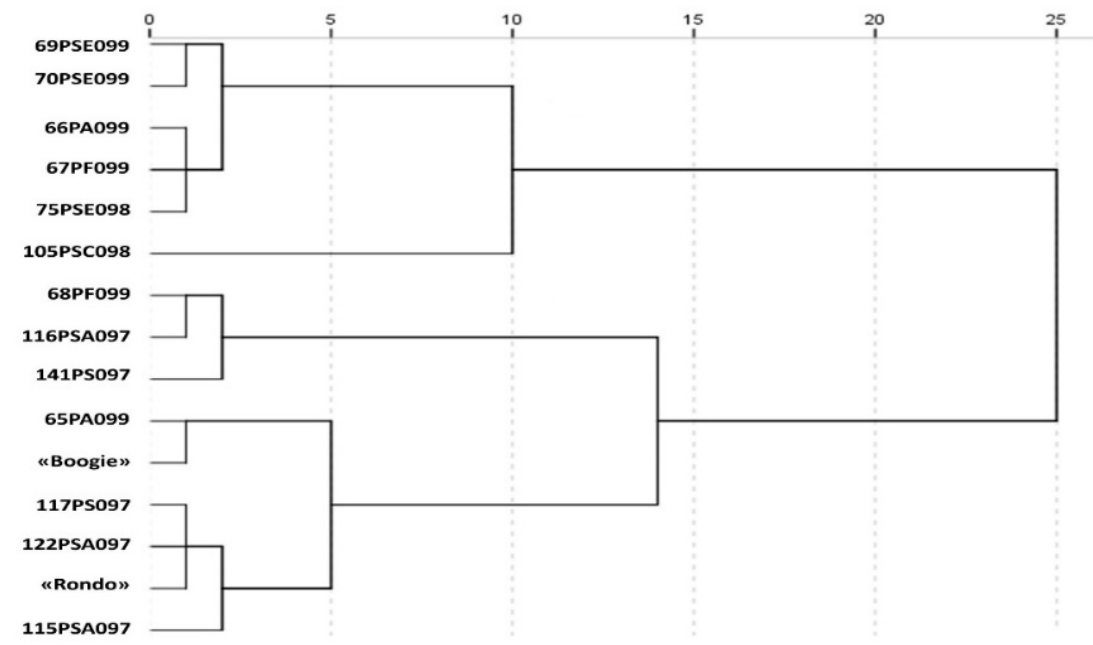

Fig. 2. Cluster analysis of individual genotypes based on leaf and stem morphology.

The 15 accessions formed two clusters at the average taxonomic distance of 142,86 considering whole morphology (Fig. 5). The largest distance was 505,06 between 65PA099 and 68PF099 while the least was 6,30 between 117PS097 and "Boogie". When considering the whole morphology, members of groups did not correspond in terms of taxonomic classification. For example, one of three $P$. sativum var. arvense took place in group II while the others were in group I. Similarly, P. sativum var. sativum accessions were divided into two separate groups.

The measurement of the phenotypical traits can provide a convenient technique for quantifying genetic similarity while at the same time defining genotype performance under relevant growing environments (Shuaib et al. 2007). The present research provided significant

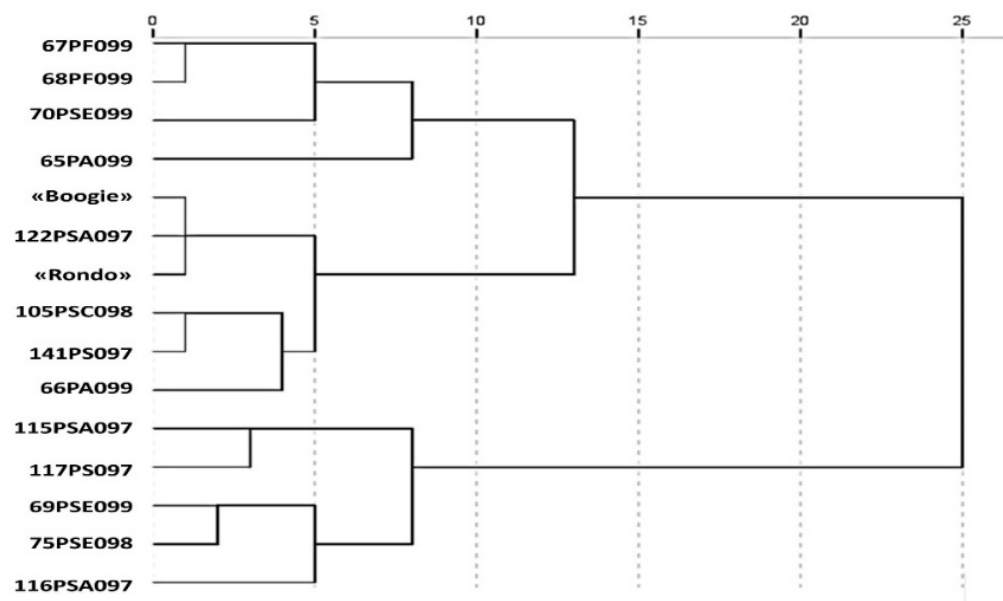

Fig. 3. Cluster analysis of individual genotypes based on flower morphology. 


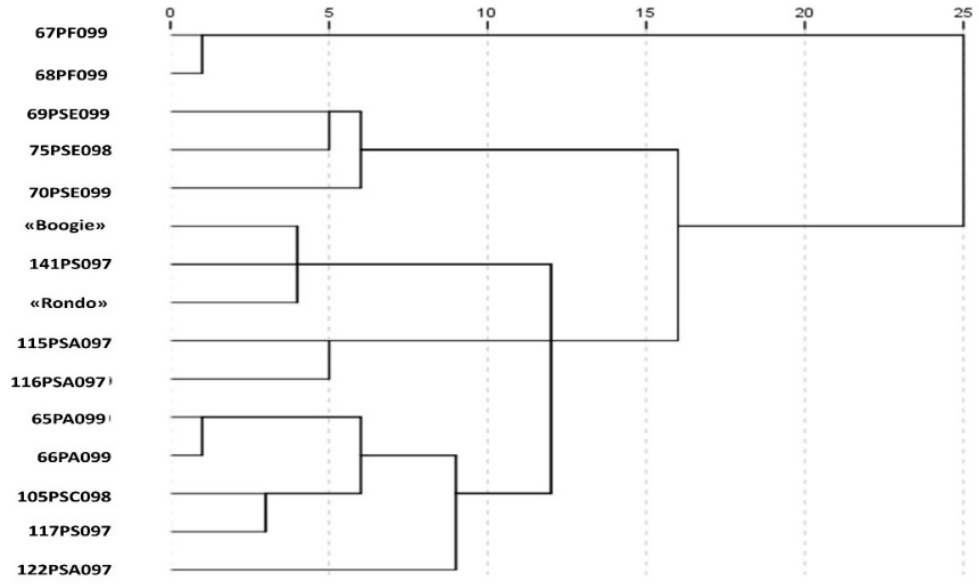

Fig. 4. Cluster analysis of individual genotypes based on seed and pod morphology.

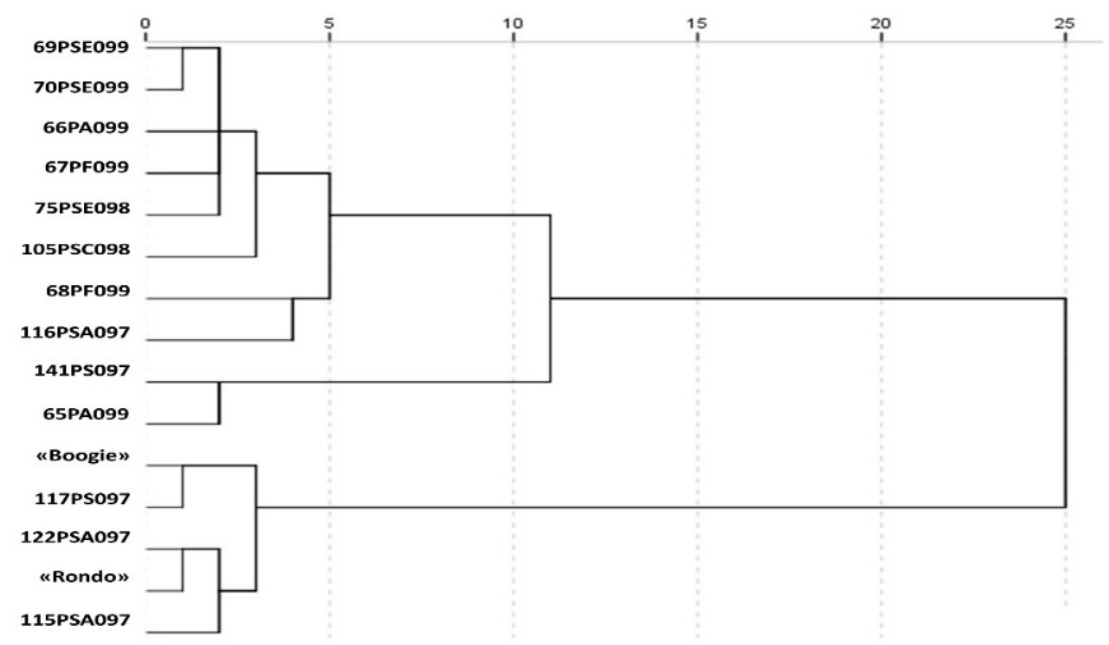

Fig. 5. Cluster analysis of individual genotypes based on whole morphology.

information in genetic variability of different Pisum accessions. In the present study, the results indicated that, when considering only flower characters and all morphological characters, Pisum accessions show clustering pattern based on the taxonomic groups. However, in clustering analysis according to the only leaf-stem characters or only seed-pod characters, accessions did not show any clustering pattern based on the taxonomic groups.

To examine the genetic relationships among different Pisum accessions, a dendrogram was performed using molecular and morphologic data together (Fig. 6). Pisum accessions were clustered into two main groups. The first group included two commercial varieties: 'Rondo' and 'Bogie'. This group includes all P. sativum var. arvense (115PSA097, 116PSA097, 105PSC098, and 122PSA097) and all P. sativum var. sativum (117PS097 and 141PS097) accessions. The combination of commercial varieties and these accessions was an expected result. However, 
according to only molecular markers results, commercial varieties were clustered together with wild forms (Fig. 1). The second group included accessions of $P$. fulvum L., P. abyssinicum L., $P$. sativum var. elatius $\mathrm{L}$.

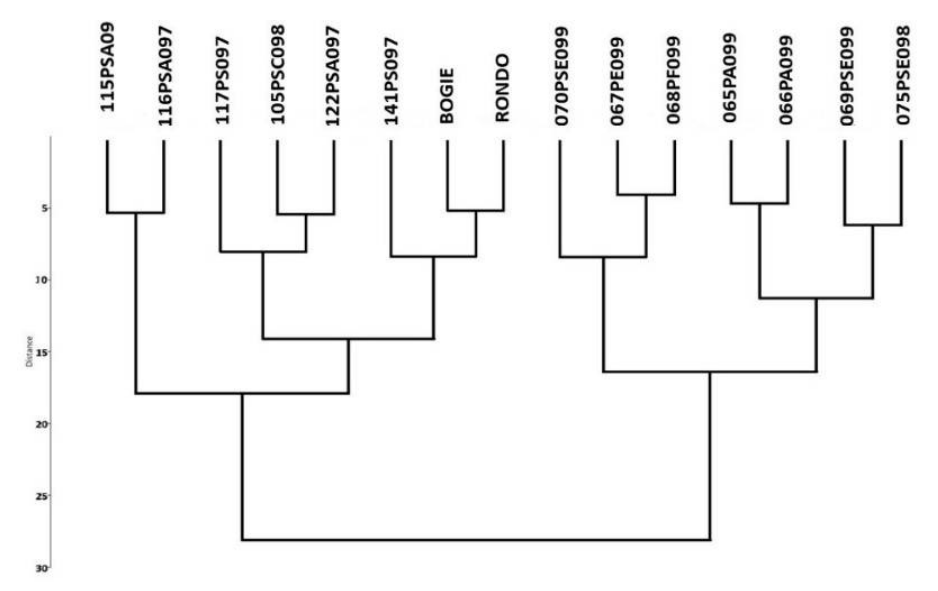

Fig. 6. Cluster analysis of Pisum genotypes based on molecular and morphological data.

In all, 15 accessions belonging to five different Pisum taxonomic groups originated from different geographic regions were analyzed using 14 SSR markers and 50 morphologic traits. Even though the number of accessions examined was restricted, the genetic variation of the samples investigated was very high as revealed by a large number of alleles, and polymorphism information content values, compared to previous studies. A broad range of diversity was also determined among morphological traits, primarily flower, seed/pod, leaf and stem.

\section{Acknowledgments}

The authors thank the authorities of Western Regional Plant Introduction Station, Pullman (USA), John Innes Centre (UK), Plant Gene Bank of the Aegean Agricultural Research Institute (TR), and "ALFA Tohum" Seed Company for providing Pisum seeds. This work is the part of Project No: TAGEM/BBAD/ 16/A09/P05/05, financed by General Directorate of Agricultural Research and Policies, Ministry of the Food, Livestock, and Agriculture, the Republic of Turkey.

\section{References}

Ahmad S, Singh M, Lamb-Palmer ND, Lefsrud M and Singh J 2012. Assessment of genetic diversity in 35 Pisum sativum accessions using microsatellite markers. Can. J. Plant Sci. 92: 1075-1081.

Cupic T, Tucak M, Popovic S, Bolaric S, Grljusic S and Kosumplik V 2009. Genetic diversity of pea (Pisum sativum $\mathrm{L})$ genotypes assessed by pedigree, morphological and molecular data. J. Food Agric. Environ. 7(3,4):343-348.

Davis PH 1970. Pisum L. In: Flora of Turkey and the East Aegean Islands, Vol. 3 (ed. P. H. Davis). pp. 370373. Edinburgh University Press, Edinburgh.

Ford R, Roux KL, Itman C, Brouwer JB and Taylor PWJ 2002. Diversity analysis and genotyping in Pisum with sequence-tagged microsatellite site (STMS) primers. Euphytica 124: 397-405.

Garcia-Valle S, Palau J and Romeu A 1999. Horizontal gene transfer in glycosyl hydrolases inferred from codon usage in Escherichia coli and Bacillus subtilis. Mol Biol Evol. 16: 1125-1134. 
Ghixari B, Vrapi H and Hobdari V 2014. Morphological characterization of pea (Pisum sativum L) genotypes stored in Albanian genebank. Albanian J. Agric. Sci (special edition). 1:164-173.

Hagenblad J, Bostrom E, Nygards L and Leino M 2014. Genetic diversity in local cultivars of garden pea Pisum sativum L. conserved on farm and in historical collections. Genet. Resour. Crop Ev. 61: 413-422.

Hildebrand CE, Torney DC and Wagner RP 1992. Informativeness of polymorphic DNA markers. Los Alamos Science 20: 100-102.

ITIS, Integrated Taxonomic Information System 2018. Integrated Taxonomic Information System on-line database. http://www.itis.gov. (accessed 09 June 2018).

Korsterin OE and Bogdanova VS 2008. Relationship of wild and cultivated forms of Pisum L. as inferred from an analysis of three markers, of the plastid, mitochondrial and nuclear genomes. Genet. Resour. Crop Evol. 55: 735-755.

Kumari P, Basal N, Singh AK, Rai VP, Srivastava CP and Singh PK 2011. Genetic diversity studies in pea (Pisum sativum L.) using simple sequence repeat markers. Genet. and Mol. Res. 12: 3540-3550.

Kupicha FK 1981. Tribe 21, Vicieae. In: Polhill RM, Raven PH (Eds.), Advances in Legume Systematics, pp. 377-381. Royal Botanic Gardens, Kew.

Loridon K, McPhee K, Morin J, Dubreuil P, Pilet-Nayel ML, Aubert G, Rameau C, Baranger A, Coyne C, Lejeune-Hènaut I and Burstin J 2005. Microsatellite marker polymorphism and mapping in pea (Pisum sativum L.). Theor. Appl. Genet. 111: 1022-1031.

Martin-Sanz A, Caminero C, Jing R, Flavell AJ and Perez-Vega M 2011. Genetic diversity among Spanish pea (Pisum sativum L.) landraces, pea cultivars and the World Pisum sp. core collection assessed by retrotransposon-based insertion polymorphisms (RBIPs). Spanish Journal of Agricultural Research 9(1): 166-178

Maxted N and Ambrose M 2001. Peas (Pisum L.) Plant genetic resources of legumes in the Mediterranean. Kluwer Academic Publishers. The Netherlands.

Nisar M, Khan A, Wadood SF, Shah AA and Hanci F 2017. Molecular characterization of edible pea through EST-SSR markers. Turk. J. Bot. 41: 338-346.

Samec P and Našinec V 1996. The use of RAPD technique for the identification and classification of Pisum sativum L. genotypes. Euphytica. 89: 229-234.

Shuaib M, Alam A, Zahir A, Waqar A, Taufiq A and Ikhtiar K 2007. Characterization of wheat varieties by seed storage protein electrophoresis. Afr. J. Biotechnol. 6: 497- 500

Teshome A, Bryngelsson T, Dagne K and Geleta M 2015. Assessment of genetic diversity in Ethiopian field pea (Pisum sativum L.) accessions with newly developed EST-SSR markers. BMC Genet. 16: 102.

Vershinin AV, Allnutt TR, Knox MR, Ambrose MJ and Ellis THN 2003. Transposable elements reveal the impact of introgression, rather than transposition, in Pisum diversity, evolution, and domestication. Mol. Biol. Evol. 20: 2067-2075. 